\title{
Nonlocal multipoint problem for a differential equation of $2 n$-th order with operator coefficients
}

\author{
Baranetskij Ya.O. ${ }^{1}$, Demkiv I.I. ${ }^{1}$, Solomko A.V. ${ }^{2, 凶}$, Sus' O.M. ${ }^{3}$ \\ In the article, the spectral properties of a multipoint problem for a differential operator equation \\ of order $2 n$ are studied. The operator of the problem has an infinite number of multiple eigenvalues. \\ Each multiple eigenvalue corresponds to a finite set of root functions. A commutative group of \\ transmutation operators is constructed. Each element of the group corresponds to the isospectral \\ perturbation of the problem operator with antiperiodic conditions. The conditions for the existence \\ and uniqueness of the solution are established for the selected family of multipoint problems, and \\ this solution is constructed too. \\ Key words and phrases: multipoint problem, antiperiodic boundary condition, root function, \\ method of transmutation operators, Riesz basis.

\footnotetext{
${ }^{1}$ Lviv Polytechnic National University, 12 Bandera str., 79013, Lviv, Ukraine

2 Vasyl Stefanyk Precarpathian National University, 57 Shevchenka str., 76018, Ivano-Frankivsk, Ukraine

${ }^{3}$ Pidstryhach Institute for Applied Problems of Mechanics and Mathematics, 3b Naukova str., 79060, Lviv, Ukraine

$\checkmark$ Corresponding author

E-mail: baryarom@ukr. net (Baranetskij Ya.O.), i hor.i.demkiv@lpnu.ua (Demkiv I.I.),

ansolvas@gmail.com (Solomko A.V.), sus@lms. Iviv.ua (Sus' O.M.)
}

\section{Introduction}

The foundations of the theory of boundary value problems for differential-operator equations are investigated in the works of J.-L. Lions and E. Magenes [18], V.L. Gorbachuk and M.L. Gorbachuk [12], S.Ya. Yakubov [24], A.A. Dezin [9].

Nonlocal problems on a finite interval for differential equations with unbounded operator coefficients were studied by Ya.O. Baranetskij et al. [3], Yu.A. Dubinskii [10], P.I. Kalenyuk et al. [16], F.E. Lomovtsev et al. [19,20], K.S. Mamedov [21], V.K. Romanko [22], S.Ya. Yakubov et al. [23] and many others.

The basics of the theory of transmutation operators are set out in the paper of V.V. Katrakhov and S.M. Sitnik [17]. The properties of differential operators that have an infinite number of multiple eigenvalues have been studied in the papers [4,6,9,13-15].

In the works $[1,2,4,5,7,15,16]$ the properties of operators spectrum are investigated by the methods of the theory of transmutation operators.

This paper is a continuation of the research $[6,7]$ for the case of nonhomogeneous boundary conditions. 


\section{Basic designations and statement of the problem}

For our investigation we will use the following notations. Let $H$ be separable Hilbert space and $A: H \rightarrow H$ be closed unbounded linear operator with discrete spectrum

$$
\sigma_{p}(A)=\left\{\mu_{k}: \mu_{k} \sim a k^{\alpha}, k \rightarrow \infty, a, \alpha>0\right\}
$$

Let $V(A)=\left\{v_{k} \in H: k=1,2, \ldots\right\}$ be the system of eigenfunctions, which forms the orthonormal basis in the space $H$ and

$$
\begin{gathered}
H\left(A^{m}\right):=\left\{v \in H, A^{m} v \in H, m \in \mathbb{R}, m \geq 0\right\}, \quad H\left(A^{0}\right)=H, \\
\|u\|_{H\left(A^{m}\right)}^{2}:=\left(A^{m} u ; A^{m} u\right)_{H}, \quad(u ; u)_{H\left(A^{m}\right)}:=\left(A^{m} u ; A^{m} u\right)_{H}, \\
L_{2}((0,1), H):=\left\{v(t):(0,1) \rightarrow H,\|v(\cdot)\|_{H} \in L_{2}(0,1)\right\}, \\
(u(t) ; v(t))_{L_{2}((0,1), H)}:=\int_{0}^{1}(u(t), \bar{v}(t))_{H} d t .
\end{gathered}
$$

Let $\left[\left(H\left(A^{m}\right) ; H\left(A^{q}\right)\right]\right.$ be an algebra of bounded linear operators $A: H\left(A^{m}\right) \rightarrow H\left(A^{q}\right)$, $D_{t}: L_{2}((0,1), H) \rightarrow L_{2}((0,1), H)$ be a strong derivative in the space $L_{2}((0,1), H)$,

$$
\begin{gathered}
W_{2}^{2 n}((0,1), A):=\left\{v \in L_{2}((0,1), H): A^{2 n} v, D_{t}^{2 n} v \in L_{2}((0,1), H),\right. \\
(u ; v)_{W_{2}^{2 n}((0,1), H)}:=(v ; u)_{L_{2}((0,1), H)}+\left(A^{2 n} v ; A^{2 n} u\right)_{L_{2}((0,1), H)}+\left(A^{2 n} v ; A^{2 n} u\right)_{L_{2}((0,1), H)}, \\
\left.\|y\|_{W_{2}^{2 n}((0,1), A)}^{2}:=\|y\|_{L_{2}((0,1), H)}^{2}+\left\|A^{2 n} y\right\|_{L_{2}((0,1), H)}^{2}+\left\|D_{t}^{2 n} y\right\|_{L_{2}((0,1), H)}^{2}\right\} .
\end{gathered}
$$

Let us consider the multipoint problem

$$
\begin{aligned}
L\left(-D_{t}^{2}, A^{2}\right) u & :=\sum_{r=0}^{n}(-1)^{r} \beta_{r} A^{2 n-2 r} D_{t}^{2 r} u(t)=f(t), \quad t \in(0,1), \\
\ell_{j} u & :=D_{t}^{2 j-2} u(0)+D_{t}^{2 j-2} u(1)=\varphi_{j}, \\
\ell_{n+j} u & :=D_{t}^{2 j-1} u(0)+D_{t}^{2 j-1} u(1)+\ell_{n+j}^{1} u=\varphi_{n+j}, \\
\ell_{n+j}^{1} u & :=\sum_{q=0}^{\chi} b_{q} D_{t}^{2 j-1} u\left(t_{q}\right),
\end{aligned}
$$

where $0=t_{0}<t_{1}<\ldots<t_{\chi}=1, \beta_{r} \in \mathbb{R}, b_{q} \in \mathbb{R}, q=0,1, \ldots, \chi, j=1,2, \ldots, n, r=0,1, \ldots, n$.

The function $u \in W_{2}^{2 n}((0,1), H)$ is called the solution of the problem (1)-(2) if

$$
\left\|L\left(-D_{t}^{2}, A^{2}\right) u-f\right\|_{L_{2}((0,1), H)}=0, \quad\left\|\ell_{j} u-\varphi_{j}\right\|_{H\left(A^{m_{j}}\right)}=0, \quad\left\|\ell_{n+j} u-\varphi_{n+j}\right\|_{H\left(A^{\left.m_{n+j}\right)}\right.}=0,
$$

with $m_{j}=2 n-2 j+\frac{3}{2}, m_{n+j}=2 n-2 j-\frac{1}{2}, j=1,2, \ldots, n$. 
Let us consider the following assumptions and theorems, that are necessary for further investigation.

Assumption $P_{1}: t_{q}=1-t_{\chi-q}, b_{q}=-b_{\chi-q}, q=0,1, \ldots, \chi$.

Assumption $P_{2}: L\left(-\omega^{2}, 1\right)=\prod_{j=1}^{n}\left(\omega^{2}-\omega_{j}^{2}\right), \operatorname{Re} \omega_{1}<\operatorname{Re} \omega_{2}<\ldots<\operatorname{Re} \omega_{n}<0, \operatorname{Re} \omega_{j}^{2}>0$.

Assumption $P_{3}: \varphi_{s} \in H\left(A^{m_{s}}\right), s=1,2, \ldots, 2 n$.

Consider the operator

$$
\begin{aligned}
L u & :=\sum_{r=0}^{n}(-1)^{r} \beta_{r} A^{2 n-2 r} D_{t}^{2 r} u, \quad u \in D(L), \\
D(L) & :=\left\{u \in W_{2}^{2 n}((0,1), H): \ell_{m} u=0, m=1,2, \ldots, 2 n\right\} .
\end{aligned}
$$

The following statements are the main results of the paper.

Theorem 1. Let the Assumptions $P_{1}, P_{2}$ hold. Then the operator $L$ has the system of root functions $V(L)$, which is the Riesz basis in the space $L_{2}((0,1), H)$.

Theorem 2. Let the Assumptions $P_{1}-P_{3}$ hold. Then for arbitrary $\beta_{r}, b_{s} \in \mathbb{R}, f \in L_{2}((0,1), H)$, $\varphi_{j} \in H\left(A^{m_{j}}\right), j=1,2, \ldots, 2 n$, the unique solution $u \in W_{2}^{2 n}(G)$ of the problem (1)-(2) exists.

Our research is structured as follows. In Section 3 the spectral properties of the problem with antiperiodicity conditions are studied. In Section 4 the solution of the problem with homogeneous conditions is constructed. In Section 5 the properties of the operator for a multipoint problem are investigated and a commutative group of transmutation operators is constructed. In Section 6 the Theorem 1 is proved. In Section 7 the solution of the problem with nonhomogeneous conditions of antiperiodicity is constructed. In Section 8 the solution of the problem with nonhomogeneous multipoint conditions is constructed and the Theorem 2 is proved for this case.

\section{The spectral problem with antiperiodic boundary conditions for diffe- rential-operator equation}

Let us consider the partial case of the problem (1)-(2) for $\beta_{r}=0, b_{q}=0, r=0,1, \ldots, n$, $q=0,1, \ldots, x$ :

$$
\begin{gathered}
L\left(-D_{t}^{2}, A^{2}\right) w_{0}:=\sum_{r=0}^{n}(-1)^{r} \beta_{r} A^{2 n-2 r} D_{t}^{2 r} w_{0}(t)=f(t), \\
\ell_{0, j} w_{0}:=D_{t}^{2 j-2} w_{0}(0)+D_{t}^{2 j-2} w_{0}(1)=0, \\
\ell_{0, n+j} w_{0}:=D_{t}^{2 j-1} w_{0}(0)+D_{t}^{2 j-1} w_{0}(1)=0, \quad j=1,2, \ldots, n .
\end{gathered}
$$

Consider the operator $L_{0}: L_{2}(0,1) \rightarrow L_{2}(0,1)$ of problem (3)-(4):

$$
\begin{aligned}
L_{0} w_{0} & :=L\left(-D_{t}^{2}, A^{2}\right) w_{0}, \quad w_{0} \in D\left(L_{0}\right), \\
D\left(L_{0}\right) & :=\left\{w_{0} \in W_{2}^{2 n}((0,1), H): \ell_{0, s} w_{0}=0, s=1,2, \ldots, 2 n\right\} .
\end{aligned}
$$


Lemma 1. The operator $L_{0}$ has the discrete spectrum

$$
\sigma\left(L_{0}\right):=\left\{\lambda_{m, q} \in \mathbb{R}: \lambda_{m, q}:=\sum_{r=0}^{n} \beta_{r} \mu_{m}^{2 n-2 r} \rho_{q}^{2 r}, \rho_{q}=\pi(2 q-1), m, q=1,2, \ldots\right\}
$$

and the system of the eigenfunctions $V\left(L_{0}\right)$, which forms the orthonormal basis in the space $L_{2}((0,1), H)$.

Proof. Consider the spectral problem

$$
\begin{aligned}
L\left(-D_{t}^{2}, A^{2}\right) w_{0}:= & \sum_{r=0}^{n}(-1)^{r} \beta_{r} A^{2 n-2 r} D_{t}^{2 r} w_{0}(t)=\lambda w_{0}(t), \\
\ell_{0, j} w_{0}:= & D_{t}^{2 j-2} w_{0}(0)+D_{t}^{2 j-2} w_{0}(1)=0, \\
\ell_{0, n+j} w_{0}:= & D_{t}^{2 j-1} w_{0}(0)+D_{t}^{2 j-1} w_{0}(1)=0, \\
& \lambda \in \mathbb{C}, j=1,2, \ldots, n .
\end{aligned}
$$

We can find the solution of the spectral problem (5), (6) as the product

$$
w_{0}(t)=y(t) v_{m}, \quad v_{m} \in V(A), m \in \mathbb{N} .
$$

To determine the unknown function $y \in W_{2}^{2 n}(0,1)$ we obtain the spectral problem

$$
\begin{aligned}
& \sum_{r=0}^{n}(-1)^{r} \beta_{r} \mu_{m}^{2 n-2 r} y^{(2 r)}(t)=\lambda y(t), \quad \lambda \in \mathbb{C}, \\
& l_{0, j} y:=y^{(2 j-2)}(0)+y^{(2 j-2)}(1)=0, \\
& l_{0, n+j} y:=y^{(2 j-1)} y(0)+y^{(2 j-1)}(1)=0, \quad j=1,2, \ldots, n .
\end{aligned}
$$

Let $L_{0, m}$ be an operator of the problem (7):

$$
\begin{aligned}
L_{0, m} y & :=\sum_{r=0}^{n}(-1)^{r} \beta_{r} \mu_{m}^{2 n-2 r} y^{(2 r)}(t), \quad y \in D\left(L_{0, m}\right), \\
D\left(L_{0, m}\right) & :=\left\{y \in W_{2}^{2 n}(0,1): l_{0, p} y=0, p=\overline{1,2 n}\right\} .
\end{aligned}
$$

Let us consider boundary value problem

$$
\begin{aligned}
& -z^{(2)}(t)=h(t), \quad t \in(0,1) \\
& z^{(r)}(0)+z^{(r)}(1)=0, \quad r=0,1 .
\end{aligned}
$$

Let $B_{0}$ be an operator of problem (8):

$$
\begin{aligned}
& B_{0} z(t):=-z^{(2)}(t), \quad z(t) \in D\left(B_{0}\right), \\
& D\left(B_{0}\right):=\left\{z \in W_{2}^{2}(0,1): z^{(r)}(0)+z^{(r)}(1)=0, \quad r=0,1\right\} .
\end{aligned}
$$

It is well known (see $[6,21])$, that the operator $B_{0}$ has the point spectrum $\sigma\left(B_{0}\right):=\left\{\phi_{q} \in \mathbb{R}\right.$ : $\left.\phi_{q}=\pi^{2}(2 q-1)^{2}, q \in \mathbb{N}\right\}$ and the system of eigenfunctions

$$
T:=\left\{\tau_{s, q}(t) \in L_{2}(0,1): \tau_{0, q}(t)=\sqrt{2} \sin (2 q-1) \pi t, \tau_{1, q}(t)=\sqrt{2} \cos (2 q-1) \pi t, q=1,2, \ldots\right\} .
$$


By direct substitution we obtain $L_{0, m}=\sum_{r=0}^{n} \beta_{r} \mu_{m}^{2 n-2 r} B_{0}^{r}$. Therefore, the operator $L_{0, m}$ has the point spectrum

$$
\sigma\left(L_{0, m}\right):=\left\{\lambda_{m, q}:=\sum_{r=0}^{n} \beta_{r} \mu_{m}^{2 n-2 r} \phi_{q}^{r}, m=\overline{1, \infty}\right\}
$$

and the system of eigenfunctions $T$.

Consider the orthonormal basis of space $L_{2}((0,1), H)$ :

$$
V\left(L_{0}\right):=\left\{v_{s, q, m}\left(t, L_{0}\right) \in L_{2}((0,1), H): v_{s, q, m}\left(t, L_{0}\right):=\tau_{s, q}(t) v_{m}, s=0,1, q, m=1,2, \ldots\right\} .
$$

From the definition of operator $L_{0}$ we have equality

$$
L_{0} v_{s, q, m}\left(t, L_{0}\right)=\lambda_{q, m} v_{s, q, m}\left(t, L_{0}\right), \quad s=0,1, q, m=1,2, \ldots
$$

The space $L_{2}((0,1), H)$ is isomorphic to the Hilbert tensor product of spaces $L_{2}(0,1)$ and $H$. Then $D\left(L_{0}\right)=\left\{w_{0} \in D\left(A^{2 n}\right) \otimes D\left(B_{0}^{n}\right)\right\} \subset L_{2}((0,1), H)$. Therefore, the operator $L_{0}$ can be represented as a polynomial $L_{0}=\sum_{r=0}^{n} \beta_{r} A^{2 n-2 r} B_{0}^{r}$ from the operators $A$ and $B_{0}$.

Taking into account the orthonormality of the system $V\left(L_{0}\right)$ in space $L_{2}((0,1), H)$, we obtain the statement of the lemma.

\section{The boundary value problem with homogeneous conditions}

Let us consider the problem (3)-(4).

Theorem 3. Let the Assumption $P_{2}$ holds. Then for arbitrary function $f \in L_{2}((0,1), H)$ the unique solution $w_{0} \in W_{2}^{2 n}((0,1), H)$ of the problem (3)-(4) exists.

Proof. We expand the functions $w_{0}(t), f(t)$ into the series with respect to the system $V\left(L_{0}\right)$ :

$$
\begin{gathered}
w_{0}=\sum_{m, q=1}^{\infty} \sum_{s=0}^{1} w_{0, s, q, m} v_{s, q, m}\left(t, L_{0}\right), \quad f=\sum_{m, q=1}^{\infty} \sum_{s=0}^{1} f_{s, q, m} v_{s, q, m}\left(t, L_{0}\right), \\
f_{s, q, m}=\left(f ; v_{s, q, m}\left(t, L_{0}\right)\right)_{L_{2}((0,1), H),} s=0,1, q, m=1,2, \ldots,
\end{gathered}
$$

where $V\left(L_{0}\right):=\left\{v_{s, q, m}\left(t, L_{0}\right) \in L_{2}((0,1), H), s=0,1, q, m=1,2, \ldots\right\}$ is the system of eigenfunctions of the operator, which is orthonormal basis of the space $L_{2}((0,1), H)$.

Substituting into the equation (3) and taking into account the completeness of the system $V\left(L_{0}\right)$, we obtain the equality $w_{0, s, q, m}=\lambda_{m, q}^{-1} f_{s, q, m}, s=0,1, q, m=1,2, \ldots$

From Assumption $P_{2}$ we have the following inequality

$$
\left|w_{0, s, q, m}\right| \leq C_{1}\left|f_{s, q, m}\right|, \quad 0<C_{1}<\infty, \quad s=0,1, q, m=1,2 \ldots
$$

Therefore

$$
\left\|w_{0}\right\|_{L_{2}((0,1), H)} \leq C_{1}\|f\|_{L_{2}((0,1), H)} .
$$

Show that $w_{0}(t) \in W_{2}^{2 n}((0,1), H)$. Taking into account the theorem on intermediate derivatives $[6,14,17]$, we should show that $A^{2 n} w_{0}(t) \in L_{2}((0,1), H)$ and $D_{t}^{2 n} w_{0}(t) \in L_{2}((0,1), H)$. 
Let $y_{1}(t)=A^{2 n} L_{0}^{-1} f(t), y_{2}(t)=B_{0}^{n} L_{0}^{-1} f(t)$. The operators $A^{2 n} L_{0}^{-1}, B_{0}^{n} L_{0}^{-1}$ have the system $V\left(L_{0}\right)$ of eigenfunctions and corresponding eigenvalues $\chi_{1, m, q}=\mu_{q}^{2 n} \lambda_{m, q}^{-1}, \chi_{2, m, q}=\phi_{q}^{n} \lambda_{m, q}^{-1}$, $m, q=1,2, \ldots$ Taking into account the Assumption $P_{2}$, we obtain the estimates

$$
\left|\chi_{s, m, q}\right| \leq C_{2}<\infty, \quad s=1,2, m, q=1,2, \ldots
$$

Therefore, $y_{s}(t) \in L_{2}((0,1), H), s=1,2$. Then from the definition of the norm in space $W_{2}^{2 n}((0,1), H)$ we obtain

$$
w_{0}(t) \in W_{2}^{2 n}((0,1), H) \quad \text { and } \quad\left\|w_{0}\right\|_{W_{2}^{2 n}((0,1), H)} \leq C_{3}\|f\|_{L_{2}((0,1), H)}, \quad 0<C_{3}<\infty .
$$

The theorem is proved.

Remark 1. From the statement of the Theorem 3 we have the following relation

$$
L_{0}^{-1} \in\left[L_{2}((0,1), H) ; W_{2}^{2 n}((0,1), H)\right] .
$$

\section{The spectral boundary problem for a differential-operator equation and transmutation operators}

Let us consider boundary value problem

$$
\begin{aligned}
-z^{(2)}(t) & =g(t), \quad t \in(0,1), \\
l^{0} z & :=z(0)+z(1)=0, \\
l^{1} z & :=z^{(1)}(0)+z^{(1)}(1)+h\left(z^{(1)}(0)-z^{(1)}(1)\right)=0, \quad h \in \mathbb{R}, r=0,1 .
\end{aligned}
$$

Let $B$ be an operator of problem (10):

$$
\begin{aligned}
& B z(t):=-z^{(2)}(t), \quad z(t) \in D(B), \\
& D(B):=\left\{z \in W_{2}^{2}(0,1): l^{r} z=0,1\right\} .
\end{aligned}
$$

In the paper [5] it is proved that the operator $B$ has eigenvalues $\mu_{m}$ and the system of root functions

$$
V(B):=\left\{v_{1, q}(t, B):=\tau_{1, q}(t), v_{0, q}(t, B)=(1-h(2 t-1)) \tau_{0, q}(t), q=1,2, \ldots\right\},
$$

for which the following relations hold

$$
B v_{1, q}(t, B)=\phi_{q} v_{1, q}(t, B), \quad B v_{0, m}(t, B)=\phi_{q} v_{0, q}(t, B)+4 \rho_{q} h v_{1, q}(t, B), \quad q=1,2, \ldots
$$

Let $B^{*}$ be an operator of the conjugate problem:

$$
\begin{gathered}
-y^{(2)}(t)=g(t), \quad t \in(0,1), \\
y(0)+y(1)+h(y(1)-y(0))=0, \\
y^{(1)}(0)+y^{(1)}(1)=0, \quad r=0,1 .
\end{gathered}
$$

The system of root functions of this operator is defined by the following relations

$$
\begin{gathered}
V\left(B^{*}\right):=\left\{v_{s, q}\left(t, B^{*}\right) \in L_{2}(0,1), \quad v_{s, q}\left(t, B^{*}\right): v_{0, q}\left(t, B^{*}\right):=\tau_{0, q}(t),\right. \\
\left.v_{1, q}\left(t, B^{*}\right)=(1+h(2 t-1)) \tau_{1, q}(t), \quad q=1,2, \ldots\right\},
\end{gathered}
$$

where

$T:=\left\{\tau_{s, q}(t) \in L_{2}(0,1): \tau_{0, q}(t)=\sqrt{2} \sin (2 q-1) \pi t, \tau_{1, q}(t)=\sqrt{2} \cos (2 q-1) \pi t, q=1,2, \ldots\right\}$, and it is biorthogonal to the system $V(B)$. 
Lemma 2. For any $h \in \mathbb{R}$ the operator $B$ has the system of root functions $V(B)$, which is the Riesz basis of the space $L_{2}(0,1)$.

Proof. The system $V(B)$ has the unique biorthogonal system $B^{*}$. Therefore, $V(B)$ is complete and minimal in the space $L_{2}(0,1)$. For any $g \in L_{2}(0,1)$ we obtain the inequality

$$
\sum_{s=0}^{1} \sum_{q=1}^{\infty}\left(g ; v_{s, q}(t, B)\right)_{L_{2}(0,1)}^{2} \leq\left(1+h^{2}\right)\|g\|_{L_{2}(0,1)}^{2}<\infty .
$$

Therefore, the system of functions $V(B)$ is the Riesz basis of the space $L_{2}(0,1)$ (see [10]).

For any $\theta_{q} \in \mathbb{R}, q=1,2, \ldots$, we define the operator $B_{\theta}: L_{2}(0,1) \rightarrow L_{2}(0,1)$ as the operator, for which the root functions are defined by the following relations

$$
\begin{aligned}
& v_{1, q}\left(t, B_{\theta}\right):=\tau_{1, q}(t), \\
& v_{0, q}\left(t, B_{\theta}\right)=\left(1+\theta_{q}(2 t-1)\right) \tau_{0, q}(t), \quad q=1,2, \ldots
\end{aligned}
$$

Taking into account (11), we obtain

$$
B_{\theta} v_{1, q}\left(t, B_{\theta}\right)=\phi_{q} v_{1, q}\left(t, B_{\theta}\right), \quad B_{\theta} v_{0, q}\left(t, B_{\theta}\right)=\phi_{q} v_{0, m}\left(t, B_{\theta}\right)+4 \rho_{q} \theta_{q} v_{1, m}\left(t, B_{\theta}\right), \quad q=1,2, \ldots
$$

We denote by $\Phi_{0}(B):=\left\{B_{\theta}\right\}$ the set of operators $B_{\theta}$ having the root functions (11) and

$$
\Phi_{0, c}(B):=\left\{B_{\theta} \in \Phi_{0}(B), \theta_{q} \in \mathbb{R},\left|\theta_{q}\right| \leq C<\infty, q=1,2, \ldots\right\} .
$$

The operator, which maps the system $V(B)$ into the system $V\left(B_{\theta}\right)$ of the root functions for the operator $B_{\theta} \in \Phi_{0}(B)$, we define by the equalities

$$
R\left(B_{\theta}\right) \tau_{s, q}(t):=v_{s, q}\left(t, B_{\theta}\right), \quad s=0,1, q=1,2, \ldots,
$$

and let $R\left(B_{\theta}\right):=E+S\left(B_{\theta}\right)$.

Define $\Gamma_{0}(B):=\left\{R\left(B_{\theta}\right): L_{2}(0,1) \rightarrow L_{2}(0,1): B_{\theta} \in \Phi_{0}(B)\right\}, \Gamma_{0, c}(B):=\Gamma_{0}(B) \cap\left[L_{2}(0,1)\right]$. For any $B_{\theta} \in \Phi_{0}(B)$ we define the biorthogonal system

$$
\begin{aligned}
V\left(B_{\theta}^{*}\right):=\left\{v_{s, q}\left(t, B_{\theta}^{*}\right) \in L_{2}(0,1): v_{0, q}\left(t, B_{\theta}^{*}\right)=\tau_{0, q}(t),\right. \\
\left.\quad v_{1, q}\left(t, B_{\theta}^{*}\right)=\tau_{1, q}(t)-\sum_{s=1}^{\infty}\left(\tau_{1, s}(t) ; v_{0, q}\left(t, B_{\theta}\right)\right)_{L_{2}(0,1)} \tau_{0, q}(t), q=1,2, \ldots\right\},
\end{aligned}
$$

where

$T:=\left\{\tau_{s, q}(t) \in L_{2}(0,1): \tau_{0, q}(t)=\sqrt{2} \sin (2 q-1) \pi t, \tau_{1, q}(t)=\sqrt{2} \cos (2 q-1) \pi t, q=1,2, \ldots\right\}$.

Therefore the following Lemma 3 holds.

Lemma 3. For any sequence $\left\{\theta_{q}\right\}_{q=1}^{\infty} \subset \mathbb{R}$ the system of functions $V\left(B_{\theta}\right)$ is complete and minimal in $L_{2}(0,1)$.

Lemma 4. The system of functions $V\left(B_{\theta}\right)$ is the Riesz basis in the space $L_{2}(0,1)$ if and only if the sequences $\left\{\theta_{q}\right\}_{q=1}^{\infty}$ are bounded. 
Proof. Necessity. If the system of functions $V\left(B_{\theta}\right)$ is a Riesz basis in the space $L_{2}(0,1)$, then it is almost normalized. Taking into account (11), we obtain the inequality

$$
0<1 \leq\left\|v_{0, q}\left(t, B_{\theta}\right)\right\|_{L_{2}(0,1)}^{2}=1+\left|\theta_{q}\right|^{2} \leq C_{4}<\infty, \quad q=1,2, \ldots, C_{4}=1+\max \theta_{q}^{2} .
$$

Sufficiency. Let us consider the relations

$$
\begin{aligned}
v_{1, q}\left(t, B_{\theta}\right) & =R\left(B_{\theta}\right) \tau_{1, q}(t)=\left(1-\theta_{q}\right) \tau_{1, q}(t)+\theta_{q} B \tau_{1, q}(t), \\
\left(h ; v_{1, q}\left(t, B_{\theta}\right)\right)_{L_{2}(0,1)} & =\left(1-\theta_{q}\right)\left(h ; \tau_{1, q}\right)_{L_{2}(0,1)}+\theta_{q}\left(h ; B \tau_{1, q}\right)_{L_{2}(0,1)}, \\
\left(h ; v_{1, q}\left(t, B_{\theta}\right)\right)_{L_{2}(0,1)}^{2} & \leq 4\left(1+\theta_{q}^{2}\right)\left(h ; \tau_{1, q}\right)_{L_{2}(0,1)}^{2}+2 \theta_{q}^{2}\left(h ; v_{1, q}(t, B)\right)_{L_{2}(0,1)^{\prime}}^{2} \\
\left(h ; v_{0, q}\left(t, B_{\theta}\right)\right)_{L_{2}(0,1)}^{2} & \left.=\left(h ; \tau_{0, q}\right)\right)_{L_{2}(0,1)^{\prime}}^{2} \\
\sum_{r=0}^{1} \sum_{q=1}^{\infty}\left(\left(R\left(B_{\theta}^{*}\right) h ; \tau_{r, q}\right)_{L_{2}(0,1)}^{2}\right. & =\sum_{r=0}^{1} \sum_{q=1}^{\infty}\left(h ; v_{r, q}\left(t, B_{\theta}\right)\right)_{L_{2}(0,1)}^{2}<C_{5}\|h\|_{L_{2}(0,1)^{\prime}}^{2} \\
C_{5} & =4\left(1+\|R(B)\|_{\left[L_{2}(0,1)\right]}^{2}\right) .
\end{aligned}
$$

Therefore,

$$
R\left(B_{\theta}^{*}\right)=E+S\left(B_{\theta}^{*}\right) \in\left[L_{2}(0,1)\right], \quad R\left(B_{\theta}\right)=E+S\left(B_{\theta}\right) \in\left[L_{2}(0,1)\right] .
$$

Taking into account the Bary theorem $[8,10]$, we obtain the statement of the lemma.

We choose two arbitrary sequences $\left\{\theta_{q}^{s}\right\}_{q=1}^{\infty}, s=1,2$, and consider the product of transmutation operators $R\left(B_{\theta}^{q}\right)=E+S\left(B_{\theta}^{q}\right), q=1,2$, and inverse operator on the set $\Gamma_{0}(B)$ :

$$
R\left(B_{\theta}^{1}\right) R\left(B_{\theta}^{2}\right)=E+S\left(B_{\theta}^{1}\right)+S\left(B_{\theta}^{2}\right), \quad R\left(B_{\theta}\right)^{-1}=E-R\left(B_{\theta}\right) .
$$

Remark 2. The set $\Gamma_{0}(B)$ is an Abelian group.

Let $B_{1}$ be an operator of the problem

$$
\begin{gathered}
-z^{(2)}(t)=h(t), \quad t \in(0,1), \\
l_{1}^{0} z:=z(0)+z(1)=0, \quad l_{1}^{1} z:=z^{(1)}(0)+z^{(1)}(1)+\sum_{r=0}^{\chi} b_{r} z^{(1)}\left(t_{r}\right), \\
B_{1} z(t):=-z^{(2)}(t), \quad z(t) \in D\left(B_{1}\right), \quad D\left(B_{1}\right):=\left\{z \in W_{2}^{2}(0,1): l_{1}^{(r)} z=0,1\right\} .
\end{gathered}
$$

We define the root functions of the operator $B_{1}$ by the relations

$$
\begin{aligned}
& v_{1, q}\left(t, B_{1}\right):=\tau_{1, q}(t), \\
& v_{0, q}\left(t, B_{1}\right):=\left(1+\theta_{q}^{1}(2 t-1)\right) \tau_{0, q}(t), \quad \theta_{q}^{1}=\sum_{r=0}^{\chi} b_{r} \tau_{0, q}\left(t_{r}\right), q=1,2, \ldots .
\end{aligned}
$$

From the relations (12) we obtain the inequality

$$
\left\|v_{1, q}\left(t, B_{1}\right)\right\|_{L_{2}(0,1)}<C_{6}, \quad s=0,1, q=1,2, \ldots
$$

Therefore, taking into account the statement of the Lemma 4, we obtain the following assertion.

Lemma 5. Let Assumption $P_{1}$ holds. Then the functions $V\left(B_{1}\right)$ is the Riesz basis in the space $L_{2}(0,1)$. 


\section{The spectral problem with nonlocal conditions for differential-operator equation}

Consider the spectral problem

$$
\begin{aligned}
L\left(-D_{t}^{2}, A^{2}\right) w & :=\sum_{r=0}^{n}(-1)^{r} \beta_{r} A^{2 n-2 r} D_{t}^{2 r} w(t)=\lambda w(t), \quad t \in(0,1), \lambda \in \mathbb{C} . \\
\ell_{j} w & :=D_{t}^{2 j-2} w(0)+D_{t}^{2 j-2} w(1)=0, \\
\ell_{n+j} w & :=D_{t}^{2 j-1} w(0)+D_{t}^{2 j-1} w(1)+\ell_{n+j}^{1} w=0, \quad j=1,2, \ldots, n .
\end{aligned}
$$

Let us prove the Theorem 1. We find the solution of spectral problem (13), (14) as the product

$$
w(t)=y(t) v_{m}, \quad v_{k} \in V(A), \quad m=1,2, \ldots
$$

To determine the unknown function $y \in W_{2}^{2 n}(0,1)$ we obtain the spectral problem

$$
\begin{aligned}
& \sum_{r=0}^{n}(-1)^{r} \beta_{r} \mu_{m}^{2 n-2 r} y^{(2 r)}(t)=\lambda y(t), \lambda \in \mathbb{C}, \\
& l_{j} y:=y^{(2 j-2)}(0)+y^{(2 j-2)}(1)=0, \\
& l_{n+j} y:=y^{(2 j-1)} y(0)+y^{(2 j-1)}(1)+\sum_{s=0}^{\chi} b_{s} y^{(2 j-1)}\left(t_{s}\right)=0, \quad j=1,2, \ldots, n .
\end{aligned}
$$

Let $L_{m}$ be an operator of the problem (15):

$$
L_{m} y:=\sum_{r=0}^{n}(-1)^{r} \beta_{r} \mu_{m}^{2 n-2 r} y^{(2 r)}(t), \quad D\left(L_{m}\right):=\left\{y \in W_{2}^{2 n}(0,1): \quad l_{j} y=0, j=1,2, \ldots, n\right\}
$$

The operator $L_{m}$ has a point spectrum (9) and the system of root functions $V\left(B_{1}\right)$ of this operator is defined by relations

$$
\begin{gathered}
L_{m} v_{1, q}\left(t, B_{1}\right)=\lambda_{m, q} v_{1, q}\left(t, B_{1}\right), \quad L_{m} v_{0, q}\left(t, B_{1}\right)=\lambda_{m, q} v_{0, q}\left(t, B_{1}\right)+\xi_{q, m} v_{1, q}\left(t, B_{1}\right), \\
\xi_{q, m}:=\sum_{r=1}^{n} \beta_{r} 2 r \mu_{m}^{2 n-2 r-1} \rho_{q}^{2 r-1}, \quad q=1,2, \ldots
\end{gathered}
$$

Therefore the operator $L$ has the system of root functions

$$
V:=\left\{v_{s, q, m}(t) \in L_{2}((0,1), H): v_{s, q, m}(t):=v_{s, q}\left(t, B_{1}\right) v_{m}, s=0,1, q, m=1,2, \ldots\right\}
$$

and is represented as a polynomial $L=\sum_{r=0}^{n} \beta_{r} A^{2 n-2 r} B_{1}^{r}$ of the operators $A$ and $B_{1}$.

Taking into account that the system $V$ is the Riesz basis in space $L_{2}((0,1), H)$, we obtain the statement of the Theorem 1. 
Let us consider the problem (1), (14).

Theorem 4. Let the Assumptions $P_{1}, P_{2}$ hold. Then for arbitrary function $f \in L_{2}((0,1), H)$ a unique solution $w \in W_{2}^{2 n}((0,1), H)$ of problem (1), (14) exists.

Proof. We expand the functions $w(t), f(t)$ into a series according to the system $V(L)$ :

$$
w=\sum_{m, q=1}^{\infty} \sum_{s=0}^{1} w_{s, q, m} v_{s, q, m}(t, L), \quad f=\sum_{m, q=1}^{\infty} \sum_{s=0}^{1} f_{s, q, k} v_{s, q, m}(t, L) .
$$

Substituting this series into the equation (1) and taking into account the completeness of the system $V(L)$ we obtain equalities

$$
w_{0, q, m}=\lambda_{m, q}^{-1} f_{0, q, m}, \quad w_{1, q, m}=\lambda_{m, q}^{-1} f_{1, q, m}-\xi_{q, m} \lambda_{m, q}^{-2} f_{1, q, m}, \quad q, m=1,2, \ldots
$$

From Assumption $P_{2}$ we have the inequality $\left|w_{s, q, m}\right| \leq C_{7}\left|f_{s, q, m}\right|, C_{7}<\infty, s=0,1$, $q, m=1,2, \ldots$ Therefore,

$$
\|w\|_{L_{2}((0,1), H)} \leq C_{8}\|f\|_{L_{2}((0,1), H),} \quad 0<C_{8}<\infty .
$$

We show that $w(t) \in W_{2}^{2 n}((0,1), H)$. Taking into account the theorem on intermediate derivatives $[6,14,17]$, we should show that

$$
w^{1}(t):=A^{2 n} w(t) \in L_{2}((0,1), H), \quad w^{2}(t):=D_{t}^{2 n} w(t) \in L_{2}((0,1), H) .
$$

The operators $A^{2 n} L^{-1}$ and $D_{t}^{2 n} L^{-1}$ have the system $V(L)$ of root functions and eigenvalues $\chi_{1, m, q}=\mu_{q}^{2 n} \lambda_{m, q}^{-1}, \chi_{2, m, q}=v_{m}^{n} \lambda_{m, q}^{-1}$.

Taking into account the Assumption $P_{2}$ we obtain estimates

$$
\left|\chi_{s, m, q}\right| \leq C_{7}<\infty, \quad s=1,2, m, q=1,2, \ldots .
$$

Therefore $w^{s}(t) \in L_{2}((0,1), H), s=1,2$.

From the definition of the norm in space $W_{2}^{2 n}((0,1), H)$ we obtain

$$
\|w\|_{W_{2}^{2 n}((0,1), H)} \leq C_{8}\|f\|_{L_{2}((0,1), H)}, \quad 0<C_{8}<\infty .
$$

The theorem is proved.

Remark 3. From the statement of the Theorem 4 we obtain the following relation

$$
L^{-1} \in\left[L_{2}((0,1), H) ; W_{2}^{2 n}((0,1), H)\right] .
$$

\section{The antiperiodic problem with nonhomogeneous conditions}

For the equation

$$
L\left(-D_{t}^{2}, A\right) v_{0}:=\sum_{r=0}^{n}(-1)^{r} \beta_{r} A^{2 n-2 r} D_{t}^{2 r} v_{0}(t)=0
$$

consider the problem with nonhomogeneous antiperiodic boundary conditions

$$
\begin{aligned}
\ell_{0, j} v_{0} & :=D_{t}^{2 j-2} v_{0}(0)+D_{t}^{2 j-2} v_{0}(1)=\varphi_{j}, \\
\ell_{0, n+j} v_{0} & :=D_{t}^{2 j-1} v_{0}(0)+D_{t}^{2 j-1} v_{0}(1)=\varphi_{n+j}, \quad j=1,2, \ldots, n .
\end{aligned}
$$


Remark 4. It is well known $[11,14,17]$, in the case $\operatorname{Re} \omega<0$ we have

$$
U(\omega, A, t)=A^{\frac{1}{2}} \exp \omega A t \in\left[H, L_{2}((0,1), H)\right],
$$

and the following relations hold

$$
\begin{gathered}
D_{t}^{s} \in\left[W_{2}^{2 n}((0,1), H) ; H\left(A^{2 n-s-\frac{1}{2}}\right)\right], \quad D_{t}^{s} v \in L_{2}\left((0,1), H\left(A^{2 n-s-\frac{1}{2}}\right)\right), \\
t \in[0,1], s=1,2, \ldots, 2 n-1
\end{gathered}
$$

for any $v(t) \in W_{2}^{2 n}((0,1), H)$.

Let

$$
\begin{aligned}
Z_{p}(A, t) & =A^{-2 n+\frac{1}{2}}\left(E+\exp \omega_{p} A\right)^{-1}\left(\exp \omega_{p} A t+\exp \omega_{p} A(1-t)\right), \\
Z_{p}(A, t) & \in\left[L_{2}((0,1), H) ; W_{2}^{2 n}((0,1), H)\right], \\
Z_{n+p}(A, t) & =A^{-2 n+\frac{1}{2}}\left(E+\exp \omega_{p} A\right)^{-1}\left(\exp \omega_{p} A t-\exp \omega_{p} A(1-t)\right), \\
Z_{n+p}(A, t) & \in\left[L_{2}((0,1), H) ; W_{2}^{2 n}((0,1), H)\right], \\
p & =1,2, \ldots, n, \quad \operatorname{Re} \omega_{1}<\operatorname{Re} \omega_{2}<\ldots<\operatorname{Re} \omega_{n}<0 .
\end{aligned}
$$

Theorem 5. Let the Assumptions $P_{2}, P_{3}$ hold. Then the function

$$
v_{0}(t):=\sum_{s=1}^{2 n} Z_{s}(A, t) h_{s}
$$

is the solution of the equation (16) for any $h_{s} \in L_{2}((0,1), H), s=1,2, \ldots, 2 n$.

Proof. Consider the following relations for the functions $v_{s}(t):=Z_{s}(A, t) h_{s}$ :

$$
\begin{gathered}
A^{2 n-2 r} D_{t}^{2 r} v_{p}(t)=\omega_{p}^{2 r} A^{\frac{1}{2}}\left(E+\exp \omega_{p} A\right)^{-1}\left(\exp \omega_{p} A t+\exp \omega_{p} A(1-t)\right) \in L_{2}((0,1), H), \\
A^{2 n-2 r} D_{t}^{2 r} v_{n+p}(t)=\omega_{p}^{2 r} A^{\frac{1}{2}}\left(E+\exp \omega_{p} A\right)^{-1}\left(\exp \omega_{p} A t-\exp \omega_{p} A(1-t)\right) \in L_{2}((0,1), H), \\
p, r=1,2, \ldots, n, s=1,2, \ldots, 2 n
\end{gathered}
$$

Therefore,

$$
v_{s}(t) \in W_{2}^{2 n}((0,1), H), \quad s=1,2, \ldots, 2 n .
$$

Substituting the function (19) into the equation (16), we obtain

$$
L\left(-D_{2}^{2}, A^{2}\right) v_{0}(t)=\sum_{p=1}^{n} L\left(-\omega_{p}^{2}, 1\right) A^{2 n}\left(Z_{p}(A, t) h_{p}+Z_{n+p}(A, t) h_{n+p}\right)=0 .
$$

Substituting the function (19) into boundary conditions (17), we obtain two systems to determine the unknowns functions $h_{s}, s=1,2, \ldots, 2 n$ :

$$
\begin{gathered}
\sum_{p=1}^{n} \omega_{p}^{2 j-2} h_{p}=\gamma_{j}, \quad \gamma_{j}=\frac{1}{2} A^{m_{j}} \varphi_{j} \in H, \\
\sum_{p=1}^{n} \omega_{p}^{2 j-1} h_{p}=\gamma_{n+j}, \quad \gamma_{n+j}=\frac{1}{2} A^{m_{n+j}} \varphi_{n+j} \in H, \\
m_{j}=2 n-2 j+\frac{3}{2}, \quad m_{n+j}=2 n-2 j-\frac{1}{2}, \quad j=1,2, \ldots, n .
\end{gathered}
$$


The determinant $\Delta_{0}$ of coefficient matrix for the first system coincides with the Vandermonde determinant, which is built on numbers $\omega_{r}^{2} \neq 0, r=1,2, \ldots, n$.

The determinant $\Delta_{1}$ of coefficient matrix for the second system is determined by the formula $\Delta_{1}=\Delta_{0} \prod_{r=1}^{n} \omega_{r}^{2}$.

Taking into account $\omega_{r} \neq 0, r=1,2, \ldots, n$, we obtain that $\Delta_{0} \neq 0$ and $\Delta_{1} \neq 0$. Thus each of the systems (20) has a unique solution. Therefore the statement of theorem is proved.

Theorem 6. Let the Assumptions $P_{2}, P_{3}$ hold. Then for any $\varphi_{s} \in H\left(A^{m_{s}}\right), s=1,2, \ldots, 2 n$, a unique solution $v_{0} \in W_{2}^{2 n}((0,1), H)$ for the problem (16), (17) exists, it is determined by the ratios (18)-(20).

\section{Existence and uniqueness of the solution of the multipoint problem with nonhomogeneous conditions}

Let us consider the problem for equation

$$
L\left(-D_{t}^{2}, A\right) v:=\sum_{r=0}^{n}(-1)^{r} \beta_{r} A^{2 n-2 r} D_{t}^{2 r} v(t)=0
$$

with nonhomogeneous multipoint conditions

$$
\begin{aligned}
\ell_{j} v & :=D_{t}^{2 j-2} v(0)+D_{t}^{2 j-2} v(1)=\varphi_{j}, \\
\ell_{n+j} v & :=D_{t}^{2 j-1} v(0)+D_{t}^{2 j-1} v(1)+\ell_{n+j}^{1} v=\varphi_{n+j}, \quad j=1,2, \ldots, n .
\end{aligned}
$$

Theorem 7. Let the Assumptions $P_{1}-P_{3}$ hold. Then for any $\varphi_{j} \in H\left(A^{m_{j}}\right), j=1,2, \ldots, 2 n$, a unique solution $v \in W_{2}^{2 n}((0,1), H)$ for the problem (21), (22) exists.

Proof. The solution of the problem (21), (22) is determined by the following relations

$$
\begin{gathered}
v(t):=v^{0}(t)+v^{1}(t), \\
v^{0}(t):=\sum_{p=1}^{n} Z_{p}(A, t) g_{p}, \quad v^{1}(t):=\sum_{p=1}^{n} Z_{n+p}(A, t) g_{n+p} .
\end{gathered}
$$

To determine the unknown functions $g_{s}, s=1,2, \ldots, 2 n$, we substitute the functions (23) into multipoint conditions (22).

We obtain two systems that are solved sequentially

$$
\begin{gathered}
\sum_{p=1}^{n} \omega_{p}^{2 j-2} g_{p}=\gamma_{j}, \quad \gamma_{j}=\frac{1}{2} A^{m_{j}} \varphi_{j} \in H, \\
\sum_{p=1}^{n} \omega_{p}^{2 j-1} g_{n+p}=\gamma_{n+j}-A^{1-2 j} \ell_{n+j}^{1} v^{0}, \quad \gamma_{n+j}=\frac{1}{2} A^{m_{n+j}} \varphi_{n+j} \in H, \\
m_{j}=2 n-2 j+\frac{3}{2}, \quad m_{n+j}=2 n-2 j-\frac{1}{2}, \quad j=1,2, \ldots, n .
\end{gathered}
$$

The solutions of the systems (24) coincide. Therefore, $v^{0}(t) \in W_{2}^{2 n}((0,1), H)$ and we obtain

$$
A^{1-2 j} \ell_{n+j}^{1} v^{0}=\sum_{p=1}^{n}\left(E+\exp \omega_{p} A\right)^{-1} \sum_{r=0}^{\chi} b_{r}\left(E+\exp \omega_{p} A t_{r}\right) g_{p} \in H
$$

Therefore, the conditions of the Theorem 6 are satisfied. Taking into account the statement of the Theorem 6 , we obtain $v(t) \in W_{2}^{2 n}((0,1), H)$. 
Let us define the solution $u(t)$ of the problem (1)-(2) as the sum $w(t)+v(t)$, where $w(t)$ is the solution of the problem (3), (14) and $v(t)$ is the solution of the problem (21), (22), respectively. Taking into account the statement of the Theorem 4 and Theorem 6 , we obtain the statement of the Theorem 2.

Let $u_{0}(t)$ be the solution of the problem (1), (2) for the case $b_{s}=0, s=0,1, \ldots, \chi$. Consider the operator

$$
G: W_{2}^{2 n}((0,1), H) \rightarrow W_{2}^{2 n}((0,1), H), G u_{0}(t):=u(t) .
$$

Remark 5. Taking into account the Theorems 2, 3 and 6, we obtain

$$
G^{-1}, G \in\left[W_{2}^{2 n}((0,1), H)\right] .
$$

\section{References}

[1] Baranetskij Ya.O., Kalenyuk P.I., Kopach M.I., Solomko A.V. The nonlocal multipoint problem with Dirichlettype conditions for an ordinary differential equation of even order with involution. Mat. Stud. 2020, 54 (1), $64-78$. doi:10.30970/ms.54.1.64-78

[2] Baranetskij Ya.O., Kalenyuk P.I., Kolyasa L.I., Kopach M.I. Nonlocal multipoint problem for ordinary differential equation of even order involution. Mat. Stud. 2018, 49 (1), 80-94. doi:10.15330/ms.49.1.80-94

[3] Baranetskij Ya.O., Demkiv I.I., Ivasiuk I.Ya., Kopach M.I. The nonlocal problem for the $2 n$ differential with unbounded operator coefficients and the involution. Carpathian Math. Publ. 2018, 10 (1), 14-30. doi: 10.15330/cmp.10.1.14-30

[4] Baranetskij Ya.O., Kalenyuk P.I., Kolyasa L.I. Spectral properties of nonself-adjoint nonlocal boundary-value problems for the operator of differentiation of evenorder. Ukr. Math. J. 2018, 70, 851-865. doi:10.1007/s11253-018-1538-4

[5] Baranetskij Ya.O., Kalenyuk P.I. Boundary-value problems with Birkhoff regular but not strongly regular conditions for a second-order differential operator. J. Math. Sci.(N.Y.) 2019, 238 (2), 1-21. doi:10.1007/s10958-019-04214-z

[6] Baranetskij Ya.O., Kalenyuk P.I., Kopach M.I., Solomko A.V. The nonlocal boundary value problem with perturbations of mixed boundary conditions for an elliptic equation with constant coefficients. II. Carpathian Math. Publ. 2020, 12 (1), 173-188. doi:10.15330/cmp.12.1.173-188

[7] Baranetskij Ya.O., Kalenyuk P.I. Nonlocal multipoint problem with multiple spectrum for an ordinary (2n)th order differential equation. J. Math. Sci.(N.Y.) 2020, 246 (2), 152-169. doi:10.1007/s10958-020-04727-y

[8] Baranetskij Ya.O., Kalenyuk P.I. Nonlocal problem with multipoint perturbations of Dirichlet conditions for even-order partial differential equations with constant coefficients. J. Math. Sci.(N.Y.) 2021, 256 (4), $375-397$. doi:10.1007/s10958-021-05433-z

[9] Dezin A.A. Differential operator equations: a method of model operators in the theory of boundary value problems. Proc. Steklov Inst. Math. 2000, 229, 1-161.

[10] Dubinskii Yu.A. On some differential-operator equations of arbitrary order. Math. USSR-Sb. 1973, 19 (1), 1-21. doi:10.1070/SM1973v019n01ABEH001672

[11] Gohberg I.C., Krein M.G. Introduction to the theory of linear nonself-adjoint operators in Hilbert Space. Amer. Math. Soc., 1969.

[12] Gorbachuk V.L., Gorbachuk M.L. Boundary value problems for operator differential equations. Naukova Dumka, Kiev, 1984.

[13] Il'in V.A. Existence of a reduced system of eigen- and associated functions for a nonself-adjoint ordinary differential operator. Proc. Steklov Inst. Math. 1976, 142, 148-155.

[14] Ionkin N.I. The solution of a certain boundary value problem of the theory of heat conduction with a nonclassical boundary condition. Differ. Uravn. 1977, 13 (2), 294-304. (in Russian)

[15] Kalenyuk P.I., Baranetskij Ya.E., Nytrebych Z.N. Generalized method of the separation of variables. Naukova Dumka, Kiev, 1993. (in Russian) 
[16] Kalenyuk P.I., Baranetskij Y.O., Kolyasa L.I. A nonlocal problem for a differential operator of even order with involution. J. Appl. Anal. 2020, 26 (2), 297-307.

[17] Katrakhov V.V., Sitnik S.M. The transmutation method and boundary-value problems for singular elliptic equations. Sovrem. Mat. Fundam. Napravl. 2018, 64 (2), 211-426. doi:10.22363/2413-3639-2018-64-2-211-426 (in Russian)

[18] Lions J.-L., Magenes E. Nonhomogeneous boundary value problems and their applications. Mir, Moscow, 1971. (in Russian)

[19] Lomovtsev F.E. A boundary value problem for even-order differential equations whose operator coefficients have variable domains. Diff. Equations 1994, 8, 1310-1322.

[20] Lomovtsev F.E., Yurchuk N.J. Boundary value problems for differential operational equations with variable operational coefficient domains. Diff. Equations 1991, 27 (10), 1754-1766.

[21] Mamedov K.S. Asymptotic behavior of distribution function of eigenvalues of abstract differential operator. Math. Notes 1982, 31, 23-29. doi:10.1007/BF01146263

[22] Romanko V.K. Solvability of boundary value problems for higher order operator-differential equations. Differ. Uravn. 1978, 14 (6), 1081-1092. (in Russian)

[23] Yakubov S.Ya., Karasik B.G., Mamedov K.S. The Fredholmicity of boundary value problems for linear differentialoperator equations for higher order. Izv. Akad. Nauk Az. SSR, Ser. Fiz.-Tekh. Mat. Nauk. 1976, 2, 76-82. (in Russian)

[24] Yakubov S.Ya. Linear operator-differential equations and their applications. Elm, Baku, 1985. (in Russian)

Received 18.06.2021

Revised 04.10.2021

Баранецький Я.О., Аемків I.I., Соломко А.В., Сусь О.М. Нелокальна багатоточкова задача для диференціального рівняння порядку 2 п з операторними коефіцієнтами // Карпатські матем. публ. — 2021. — T.13, №2. — С. 501-514.

У статті вивчаються спектральні властивості багатоточкової задачі для диференціального операторного рівняння порядку $2 n$. Оператор задачі має нескінченну кількість кратних власних значень. Кожному кратному його власному значенню відповідає скінченний набір кореневих функцій. Побудована комутативна група операторів перетворення. Кожному елементу групи відповідає ізоспектральне збурення оператора задачі з антиперіодичними умовами. Аля вибраної сім'ї багатоточкових задач встановлено умови існування та єАиності розв'язку, а також цей розв' язок побудовано.

Ключові слова і фрази: багатоточкова задача, антиперіодична крайова умова, коренева функція, метод операторів перетворення, базис Рісса. 